\section{RIMCIS}

\section{Hipatia Press}

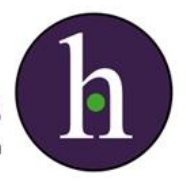

Instructions for authors, subscriptions and further details:

http://rimcis.hipatiapress.com

\title{
La Configuración Espacial de la Ciudad como Factor de Definición de la Realidad Socio-Demográfica. Análisis Electoral de un Entorno Urbano
}

Manuel Jacinto Roblizo-Colmenero ${ }^{1}$

1) University of Castilla-La Mancha, Spain

Date of publication: March $30^{\text {th }}, 2015$

Edition period: March 2015 - July 2015

To cite this article: Roblizo-Colmenero, M.J. (2015). La Configuración Espacial de la ciudad como Factor de Definición de la Realidad SocioDemográfica. Análisis Electoral de un Entorno Urbano. International and Multidisciplinary Journal of Social Sciences, 4(1), 1-22. doi:

10.17583/rimcis.2015.01

To link this article: $\underline{\text { http://dx.doi.org/10.17583/rimcis.2015.01 }}$

\section{PLEASE SCROLL DOWN FOR ARTICLE}

The terms and conditions of use are related to the Open Journal System and to Creative Commons Attribution License (CC-BY). 


\section{Spatial Configuration of City as a Factor for Definition of Sociodemographic Reality. Electoral Analysis of an Urban Setting}

Manuel Jacinto Roblizo-Colmenero Universidad de Castilla-La Mancha

\section{Abstract}

The article carries out a multidisciplinary analysis of the electoral behaviour of a particular urban setting, as a means to observe how the spatial urban configuration is depicted on the socio-demographic structure of electoral body. Covering analytical categories from the urban geography and the sociology, and arising from the social reality of the different urban areas, a case study is provided that demonstrates the existing link between some particularly relevant socio-demographic traits and the attitude that the citizens defined by them show in the ballot boxes. We observe, therefore, the socio-demographic -and, as a consequence, electoralrelevance of the urban spatial configuration. At the same time, we identify an interpretative approach to electoral behaviour that includes both the structural elements that emphasise the individual's social conditionings and those that, founded on rational action and election models, privilege the individual over the structure.

Keywords: urban space, political behaviour, city, elections 


\title{
La Configuración Espacial de la Ciudad como Factor de Definición de la Realidad Socio- Demográfica. Análisis Electoral de un Entorno Urbano
}

\author{
Manuel Jacinto Roblizo-Colmenero \\ Universidad de Castilla-La Mancha
}

\section{Resumen}

El artículo lleva a cabo un análisis interdisciplinar del comportamiento electoral de un entorno urbano específico, como fundamento para observar como la configuración espacial urbana aparece recogida en la estructura sociodemográfica del cuerpo electoral. Recogiendo categorías analíticas procedentes de la geografía urbana y la sociología, y partiendo de la realidad social de las distintas zonas urbanas de una ciudad, se aporta un estudio de caso que muestra el vínculo existente entre determinados rasgos sociodemográficos especialmente relevantes y la actitud ante las urnas de los ciudadanos definidos por ellos. Observamos, por lo tanto, el calado sociopolítico de la configuración espacial urbana, a la vez que percibimos una vertiente interpretativa del comportamiento electoral en base a conceptualizaciones sociológicas esenciales que recogen tanto a aquellos elementos estructurales que ponen el énfasis en el condicionamiento social del individuo como a aquellos otros que, basados en modelos de elección y elección racional, priman al individuo sobre la estructura.

Palabras clave: espacio urbano, comportamiento político, ciudad, elecciones 


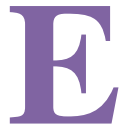

1 presente artículo se deriva de la voluntad de mostrar como la ciudad nos aporta un ingrediente explicativo de máxima utilidad para rastrear la persistencia de las diferencias sociodemográficas en la vida social, con su concreción en el comportamiento electoral. En este trabajo, hemos optado por un conjunto de variables y de procedimientos estadísticos definidos en torno a la configuración urbana del voto en una ciudad de poco más de 170.000 habitantes. Hemos utilizado, para ello, dos fuentes secundarias. La primera de ellas será el conjunto de indicadores socioeconómicos recogidos en el informe Barrios de Albacete: igualdad, situación social y recursos, publicado por el Observatorio Municipal de Igualdad de Oportunidades de Albacete. Esta fuente aporta, entre otros muchos datos, los valores estandarizados de puntuaciones $\mathrm{Z}$ (de distancias a la media en unidades de desviación típica) del conjunto de los barrios de la ciudad, calculados sobre la base de las variables envejecimiento, nivel educativo y rango socioeconómico, que hemos considerado de utilidad para nuestro análisis. La otra fuente secundaria la aportarán los resultados electorales, distribuidos por barrios y zonas urbanas, de los comicios locales del 15 de mayo de 2011; sobre la base de datos por sección, distrito y mesa, hemos hecho una agrupación por áreas de la ciudad y, a su vez, partiendo de esos datos, hemos calculado las puntuaciones $\mathrm{Z}$ de distancias a la media en unidades de desviación típica para los valores de voto PSOE, PP y participación electoral. Estos tres valores son representativos de las sensibilidades electorales básicas $y$, a su vez, pueden encontrarse numéricamente, en cada una de las áreas, en unas cantidades suficientemente significativas. Finalmente, a fin de obtener evidencia empírica estadística que sustente nuestros análisis, hemos llevado un análisis de regresión que ha puesto en relación las variables sociodemográficas indicadas con los valores de voto también señalados.

Aunque, administrativamente, las pedanías están integradas en la ciudad de Albacete, no las hemos incluido en nuestro análisis, por una parte, porque la principal razón de ser de nuestro trabajo radica en observar diferencias en comportamiento electoral en ciudadanos que comparten un mismo entorno urbano -las pedanías son pequeñas localidades distantes geográfica y socialmente de Albacete-; por otra parte, en ellas se eligen a los llamados alcaldes pedáneos de una manera diferenciada a la que se produce en la 
elección de alcaldesa y concejales de Albacete, con lo que tampoco estaríamos hablando de un mismo proceso electoral como tal. No incluimos en nuestro análisis a los barrios de Universidad, Llanos del Águila, Canal de María Cristina, San Pedro-Mortero y las Cañicas. En los tres primeros casos, porque al tratarse de barrios de reciente creación -y todavía en expansiónno es posible extraer indicadores socioeconómicos como los que tomaremos como base de nuestras reflexiones; en el caso de los dos últimos barrios, porque debido a su pequeño tamaño y a la falta de correspondencia completa entre secciones censales y barrios, no se dispone de datos del Censo de Población y de viviendas, con lo que la información de carácter socioeconómico quedaría incompleta.

Tomamos como punto de partida para este análisis los datos de las elecciones locales 2011. Dadas las pretensiones de este artículo, hubiera sido igualmente razonable utilizar los datos de los comicios autonómicos que tuvieron lugar de manera simultánea. Igualmente, las elecciones generales de noviembre de 2011 ofrecerían otra posibilidad más de uso de datos. En todo caso, dado que el nivel de análisis no se extiende más allá de un ámbito local, hemos finalmente considerado que, en la tesitura de elegir unos comicios que nos sirvieran como base, los locales resultaban ser una elección más lógica.

\section{La Configuración Sociodemográfica de los Entornos Urbanos}

El entorno ecológico urbano que ofrecen los barrios y áreas residenciales se revela, entendemos, como un referente óptimo para una mejor comprensión de los condicionantes del comportamiento electoral. Y ello es así porque hay, sin duda, sobradas razones sociológicas para continuar asumiendo la reivindicación de la Escuela de Chicago acerca del lugar, en tanto entorno urbano, como algo cargado de significatividad social. Aquellos pioneros de la sociología urbana se ocuparon en generar unas medidas, basadas empíricamente, con las que delimitar áreas urbanas socialmente definidas. Estas "áreas naturales" -por usar la propia terminología de la Escuela de Chicago- quedaban definidas en base a la distribución geográfica de grupos poblacionales concretos, como las minorías étnicas, o por la incidencia de ciertos factores sociales de connotaciones problemáticas, como las tasas de 
delincuencia. Urbanísticamente hablando, 'birds of a feather flock together', como se ha señalado en el ámbito anglosajón a través de la conocida organización en sistemas geodemográficos ACORN (A Classification of Residential Neighbourhoods):

basada en la idea de que "los pájaros del mismo plumaje forman bandada", reconoce el hecho de que gente con características similares de tipo económico, social y de estilo de vida tienden a agruparse en barrios concretos y a mostrar pautas similares de comportamiento comercial y de apariencia (Wilson, Gilligan y Pearson, 1992, p. 202) ${ }^{1}$

El característico sistema ACORN, inicialmente concebido como una forma de estructurar el entorno urbano con propósitos de mero marketing, resultó ser un mecanismo emblemático con el que se pudo apreciar que, en términos de desarrollo urbano, la clase social era un factor que todavía mostraba un alto nivel de relevancia en relación a una variedad de efectos. Ello incluía alguno tan -aparentemente- puramente biológico -y, por lo tanto, susceptible de ser considerado mínimamente influido por lo socialcomo las tasas de mortalidad y la esperanza de vida, significativamente distintas en los pequeños distritos electorales británicos (Mitchel, Dorling y Shaw, 2000).

A partir de este tipo de premisas, nosotros seguiremos la tradición marcada por la pionera Escuela de Chicago -aunque con planteamientos metodológicos ciertamente no equiparables-, en el sentido de asumir que la ciudad, por sí misma, puede ser considerada una especie de experimento social, y no como un mero asentamiento sobre el territorio. Por ello, a través de nuestros análisis, hacemos nuestra la idea de que la sociología debe "bajar a las calles" para estudiar problemas urbanos y procesos sociales electorales, más concretamente, en nuestro caso:

La ciudad, en pocas palabras, muestra lo bueno y lo malo que encontramos, en sus formas extremas, en la naturaleza humana. Es este hecho, más que ningún otro, el que justifica el punto de vista que hace de la ciudad un laboratorio o clínica en el que la naturaleza humana y los procesos sociales pueden ser conveniente $\mathrm{y}$ provechosamente estudiados (Park, 1952, p. 611-612) ${ }^{2}$ 
Nuestros análisis se sustentan, igualmente, en la perspectiva que utilizara paradigmáticamente Cox (1969) con el concepto de "superficie de respuesta de voto", que definió en base a la influencia de los efectos de grupo (o, lo que es lo mismo, de la estructura social), de vecindario, de amigos y vecinos, de relocalización inmigratoria y de protección local. Es también Kevin Cox, en esa misma obra, quien analiza el fenómeno del distance-decay, que señala como la interacción disminuye con la distancia entre los sujetos -o, a la inversa, como es más notoria entre sujetos que comparten, desde la cercanía, un espacio-, de una manera que aporta una valiosa categoría de fundamentación a nuestro trabajo, y que también recogieran tanto Mervyn Busteed (1975) como Peter Taylor y Ronald Johnson (1979). En el ámbito territorial que nos ocupa, los análisis del contexto urbano que lleva a cabo, de manera pionera, Juan de Dios Izquierdo (1984) representan un valioso precedente y punto de referencia.

En líneas muy generales, es posible sintetizar la diversidad de aportaciones explicativas acerca del comportamiento electoral en tres grandes modelos: el llamado enfoque sociológico, el de identificación de partido y el de elección racional. De manera muy característica, la primera queda representada por los conocidos trabajos de Seymour M. Lipset, para el que "más que cualquier otra cosa, la lucha de partidos es un conflicto entre clases, y el fenómeno más notable del apoyo a un partido político consiste en que virtualmente en todo país económicamente desarrollado los grupos de menores ingresos votan principalmente por partidos de izquierda, mientras que los grupos de mayores ingresos lo hacen por los de derecha" (Lipset, 1987, p. 197). Desde este punto de vista, la perspectiva elegida para este artículo vendría a representar una forma de corroborar (o descartar) el vínculo existente entre nivel socioeconómico de las distintas zonas urbanas y voto a las principales opciones. $\mathrm{O}$, en todo caso, los indicadores de regresión lineal vendrían a aportar una forma de matizar esa relación.

El llamado modelo de identificación de partido (Lewis-Beck et al., 2008; Green, Palmquist y Schickler, 2004; Harrop y Miller, 1987, p. 130 y ss) remite, esencialmente, a la transmisión de valores y preferencias partidistas entre generaciones. Sin embargo, también reflexiona acerca de en qué medida los electores, en su mayoría, adquieren una identificación duradera con una opción política. Cuando por motivos coyunturales - de los cuales 
una crisis económica de amplias dimensiones puede ser un perfecto ejemplose registra una cierta cantidad de cambio o volatilidad electoral -que incluso puede ser más que suficiente para generar un cambio de gobierno-, es posible encontrar también como el apoyo a los principales partidos se sustenta en una identificación estable fruto de la pertenencia social.

De esta manera, encontraríamos un paradigma que nos serviría para explicar las pautas de estabilidad que, aunque normalmente minusvaloradas en los análisis de estos comicios, existen de una manera estadísticamente muy representativa. Sin embargo, el cambio político -sin duda, el hecho más notoriamente destacable de los comicios de mayo del 2011- quedaría infravalorado, y es para ello que las aportaciones basadas en la lógica de la elección racional son de especial utilidad $-\mathrm{y}$, como veremos, resultan ser un paradigma especialmente fructífero para tiempos de graves convulsiones económicas-. Inevitablemente, el cálculo de beneficios basado en la valoración del pasado, tanto remoto como inmediato, ocupa un lugar central en esta amplia línea de pensamiento sociológico. Así, para Anthony Downs:

Toda elección es un juicio sobre el pasado del partido en el poder (...). A los hombres racionales no les interesa la política per se, sino sus rentas de utilidad. Si, a su juicio, las que obtienen en la actualidad son muy bajas, pueden creer que cualquier cambio previsible las aumentará. En tal caso, lo racional para ellos es votar contra el gobierno, es decir, a favor del cambio en general (Downs, 1973, p. 44-45)

De esta manera, Downs aporta -desde las premisas características de la teoría de la acción racional- unos factores que cabría denominar instrumentales.

En cuanto a la distribución diferencial de la participación en el ámbito urbano, la distinción clásica entre centro y periferia que formulara Milbrath (1965) -que usaba esa denominación con referencia a estratos sociales, y no urbanísticos, pero que en esta ocasión coincide con unas categorías útiles en la descripción espacial de la ciudad-, nos aporta un útil engranaje conceptual para el contraste, según el cual las clases socioculturalmente más desfavorecidas tienden en mayor medida a la abstención, mientras que, a la 
inversa, las clases más favorecidas resultan ser más propensas a la participación electoral.

\section{Un Análisis Electoral de Sociología Urbana: La Cartografía Social de una Ciudad}

La configuración espacial que encontramos en la ciudad de Albacete resulta ser especialmente sencilla $-\mathrm{y}$, por ello, especialmente nítida cuando se trata de observar sus rasgos sociodemográficos-. El contraste entre centro urbano y barrios periféricos responde a lo que cabría esperar en base a un análisis tradicional de lo que históricamente suelen representar esas configuraciones urbanas. A diferencias de otras ciudades, en las que la degradación de las residencias ubicadas en el centro histórico y geográfico ha determinado que los sectores de mejor nivel adquisitivo tiendan a ubicarse en localizaciones más diferenciadas, en el caso de Albacete la configuración urbana hace que el centro de la ciudad sea un indicativo nítido de clases sociales de una condición socioeconómica alta o media-alta. En ese concepto de centro cabría incluir una zona, Villacerrada, constituida por una gran manzana con una igualmente gran plaza interior, que se construyó en los años 70 sobre lo que fuera el llamado Alto de La Villa, hasta entonces habitado por sectores de población de bajo nivel económico. En la ubicación extrema en cuanto a rasgos sociodemográficos desfavorables encontramos dos barrios poblados en una buena parte por población con dificultades diversas de integración social; en ellas encontraríamos la casi totalidad de núcleos significativos de población de etnia gitana, que se ubican en otras zonas de la ciudad en una medida mucho menor. Así, el barrio de La Estrella, popularmente conocido como "El Cerrico", es la zona de ubicación histórica de la población de esta etnia -junto al citado y desaparecido Alto de La Villa-. Anexo a él se construyeron las llamadas 600 viviendas -el barrio de La Milagrosa-, un grupo de viviendas de promoción pública en el que se ubicaron familias con dificultades socioeconómicas, como se deduce del carácter social de estas construcciones.

Otro de estos barrios de viviendas sociales lo encontramos en Hermanos Falcó, popularmente llamado "Las Quinientas" por el número de estas viviendas sociales que lo integraban originariamente. Esta zona, al igual que 
Sepulcro-Bolera, Pedro Lamata, San Pedro Mortero, Santa Teresa, Vereda, San Pablo y Cañicas, integran los barrios que quedaban al otro lado de la carretera de circunvalación de la ciudad, y todos ellos estaban integrados por población de nivel socioeconómico desfavorecido, con lo que esta vía tomaba una gran relevancia como elemento de delimitación geográfica y social. La pureza de esa configuración queda difuminada cuando se comienzan a construir zonas de viviendas, en su mayor parte unifamiliares, que, como tales, tenían necesariamente que estar alejadas del denso centro urbano y que, sin embargo, eran habitadas por familias de alto nivel socioeconómico. La primera de estas configuraciones fue la zona de Universidad, todavía en crecimiento con viviendas tanto unifamiliares como bloques. Con posterioridad a ella, han aparecido nuevas ubicaciones con viviendas de características similares en cuanto a lejanía del centro -muchas de las cuales han sufrido los efectos de la crisis económica y se encuentran sin habitar- $y$, sin embargo, habitadas por población de nivel socioeconómico no necesariamente bajo. La dificultad en el acceso a la vivienda de los últimos años, que ha tenido un carácter considerablemente interclasista, empujaba a buscar precios asequibles incluso a profesionales o familias de nivel económico medio. Entre la zona del otro lado de la antigua carretera de circunvalación y el centro urbano encontramos un amplio segmento de áreas de un carácter que podríamos denominar intermedio, habitadas por lo que, grosso modo, llamaríamos clases medias. La configuración urbana tiende, pues, como vemos, a tornarse progresivamente más compleja.

En la Tabla 1 ofrecemos los datos "en bruto" de resultados electorales de utilidad para nuestro análisis. Dado que no tratamos de hacer un análisis electoral en sentido estricto, entendemos que no es necesario aportar un desglose minucioso de toda la información de resultados de los comicios que nos ocupan, sino ofrecer al lector la información de la que hemos extraído los indicadores estadísticos que aportaremos en las siguientes páginas. En la Tabla 2, por su parte, recogemos los indicadores sociológicos, en forma de puntuaciones $\mathrm{Z}$ de unidades de desviación típica, que utilizaremos para tratar de corroborar -o, en su caso, descartar o matizar- el vínculo existente entre rasgos sociodemográficos y datos electorales. 
Tabla 1.

Resultados electorales por barrios. Elecciones locales 2011

\begin{tabular}{lccccc}
\hline & \multicolumn{2}{c}{ Voto PSOE } & \multicolumn{2}{c}{ Voto PP } & Abst. \\
\hline Nombre barrio & $\%$ & Votos & $\%$ & Votos & $\%$ \\
\hline Carretas-H. Marzo & 24,55 & 1241 & 58,39 & 2951 & 27,84 \\
Centro ciudad & 20,86 & 1717 & 65,79 & 5415 & 23,78 \\
El Pilar & 39,85 & 2241 & 45,05 & 2533 & 32,16 \\
Fátima & 36,16 & 2510 & 47,38 & 3280 & 29,90 \\
Feria-El Molino & 26,12 & 1384 & 59,98 & 3178 & 25,03 \\
Franciscanos & 30,61 & 3534 & 55,25 & 6380 & 28,53 \\
Hnos. Falcó & 51,90 & 669 & 34,83 & 499 & 40,35 \\
Hospital & 34,74 & 1525 & 48,02 & 2108 & 34,33 \\
Industria-Cubas & 31,84 & 1589 & 52,31 & 2611 & 29,59 \\
La Estrella & 53,08 & 155 & 31,51 & 92 & 54,45 \\
La Milagrosa & 51,24 & 269 & 25,33 & 133 & 58,17 \\
La Pajarita & 27,31 & 604 & 57,14 & 1264 & 27,64 \\
Parque Sur & 25,08 & 1049 & 57,27 & 2395 & 24,18 \\
Pedro Lamata & 42,98 & 389 & 43,20 & 391 & 31,18 \\
Polígono San Antón & 37,08 & 1093 & 43,93 & 1295 & 26,65 \\
San Antonio Abad & 34,68 & 1002 & 46,24 & 1336 & 29,16 \\
San Pablo & 41,97 & 1646 & 42,61 & 1671 & 36,18 \\
Santa Teresa & 35,06 & 135 & 49,61 & 191 & 39,18 \\
Sepulcro-Bolera & 29,68 & 387 & 54,22 & 707 & 29,40 \\
Vereda & 35,38 & 755 & 47,38 & 1011 & 34,94 \\
Villacerrada & 22,94 & 192 & 63,08 & 528 & 20,51 \\
\hline
\end{tabular}

Fuente: elaboración propia a partir de datos por distrito, sección y mesa del Ministerio del Interior, 2011.

Los tres factores que hemos incorporado en nuestro análisis -amén de los datos electorales- quedan definidos en base a las siguientes variables: el rango socioeconómico se establece en base a las variables \% personas en hogares sin empleo, salario estimado, \% clases baja y media baja y \% personas en viviendas hipotecadas (Observatorio Municipal de Igualdad de Oportunidades de Albacete, 2011, pp. 48-52, 58-59, 72-73, 173). Por su parte, el factor nivel educativo se establece en base a la variable porcentaje de personas con bajo nivel educativo (ibídem, pp. 37-39), entendiendo por tales el porcentaje de individuos de entre 25 y 64 años de edad que han acabado, como máximo, el primer grado escolar; la variable se forma mediante la adición de las categorías "Analfabetos", "Sin estudios" y "Primer Grado" del Censo de Población y Viviendas del 2001. Finalmente, 
el envejecimiento está definido por la variable grado de juventud/vejez de cada uno de los barrios (ibídem, p. 16). Ello se hace así para evitar la simplificación que podría suponer el establecer una proporción entre población de más de 65 años sobre el total de la ciudad, por implicar la comparación matemática entre grupos que no son homogéneos.

Tabla 2.

Indicadores socioeconómicos (valores estandarizados)

\begin{tabular}{lccc}
\hline Nombre barrio & Envejecimiento & $\begin{array}{c}\text { Factor } \\
\text { educativo }\end{array}$ & $\begin{array}{c}\text { Factor } \\
\text { socioeconómico }\end{array}$ \\
\hline Carretas-H. Marzo & $-0,24$ & $-0,71$ & $-0,24$ \\
Centro ciudad & 1,05 & $-1,06$ & 0,77 \\
El Pilar & $-0,12$ & 0,17 & 0,30 \\
Fátima & 0,75 & $-0,01$ & $-0,13$ \\
Feria-El Molino & 0,49 & $-0,54$ & $-0,44$ \\
Franciscanos & 0,87 & $-0,30$ & $-0,36$ \\
Hnos. Falcó & 0,91 & 0,33 & 0,33 \\
Hospital & $-0,25$ & $-0,31$ & 0,07 \\
Industria-Cubas & $-0,59$ & $-0,50$ & $-0,14$ \\
La Estrella & $-0,83$ & 2,47 & 1,93 \\
La Milagrosa & $-2,83$ & 2,90 & 1,11 \\
La Pajarita & 1,69 & $-0,87$ & $-0,71$ \\
Parque Sur & $-0,01$ & $-0,96$ & $-0,72$ \\
Pedro Lamata & $-0,13$ & 0,36 & 0,30 \\
Polígono San Antón & 0,67 & $-0,30$ & $-0,38$ \\
San Antonio Abad & $-0,48$ & $-0,26$ & 0,14 \\
San Pablo & $-1,03$ & 0,19 & 0,33 \\
Santa Teresa & $-0,73$ & $-0,12$ & 0,46 \\
Sepulcro-Bolera & $-0,28$ & $-0,06$ & $-0,05$ \\
Vereda & $-1,53$ & $-0,04$ & $-0,03$ \\
Villacerrada & 0,32 & $-1,16$ & $-0,99$ \\
\hline
\end{tabular}

Fuente: Observatorio Municipal de Igualdad de Oportunidades de Albacete, 2011, p. 177.

Resulta importante tener en cuenta, de cara a la interpretación de los datos, que los dos primeros factores señalados -rango socioeconómico y nivel educativo- están definidos en base a valores socialmente negativos. De esta manera, el nivel educativo no se establece en base al máximo nivel alcanzado por las personas investigadas -que es una opción metodológica utilizada con cierta frecuencia- o a parámetros similares, sino que es el 


\section{Roblizo-Colmenero - La Configuración Espacial de la Ciudad}

resultado de obtener el porcentaje de individuos con bajo nivel educativo; ello implicará, en cuanto a la lectura de los resultados, que puntuaciones elevadas en este indicador -nivel educativo- significarán un alto número de individuos en niveles bajos de estudios, y en modo alguno deberá entenderse como que los niveles generales de la población de cada uno de los barrios sean elevado.

Lo mismo ocurre en el caso del rango socioeconómico, definido básicamente en base a indicadores socialmente negativos -personas en hogares sin empleo...-, por lo que una mayor puntuación en ese rango no implicará una mejor calidad de vida ni un mayor nivel de riqueza en el barrio en cuestión. La forma de cálculo de nivel de envejecimiento sí que es más fácilmente entendible intuitivamente, y mayores puntuaciones sí que representarán una población más envejecida, y a la inversa. Por ello, en nuestro artículo sustituimos las expresiones "nivel educativo" y "rango socioeconómico", que parecen indicar una idea de jerarquización que puede resultar confusa, por las expresiones "factor educativo" y "factor socioeconómico".

El contraste geográfico entre núcleos rurales/núcleos urbanos, especialmente cuando se trata de su versión más extrema, es nítido, y fácilmente traducible a una dicotomía entre conservadurismo (no ideológico, sino actitudinal) / dinamismo -correspondiendo aquél a las zonas rurales y éste a las urbanas-. El comportamiento de los sectores de mayor edad y menor nivel educativo y socioeconómico no es, sin embargo, equiparable cuando la distinción se hace en el seno de un entorno urbano. Los barrios más desfavorecidos -de menor nivel socioeconómico y cultural $\mathrm{y}$, en cierta medida, más envejecidos- muestran una notoria lealtad al PSOE, que puede incrementarse o disminuir en algunos puntos a lo largo de los sucesivos procesos electorales, pero que se revela como inquebrantable - a diferencia de lo que ocurre con los entornos desfavorecidos rurales-. En lo relativo a la participación, la constancia también es un rasgo significativo de las zonas más marginales. De hecho, históricamente barrios como La Estrella y La Milagrosa ofrecen porcentajes de abstención habitualmente por encima del $40 \%$-a veces incluso del 50\%-, aunque, curiosamente, en ambos casos en el 82 -año, recordemos, de la primera victoria socialista en el actual periodo democrático- se produjo una movilización electoral que 
situó la abstención en porcentajes de en torno al 20\%. En los comicios que ahora nos ocupan, las zonas que se sitúan en porcentajes de abstención superiores al $40 \%$ son, de nuevo, La Estrella y La Milagrosa -las "600 viviendas"- (ambas por encima del 50\%), y Hermanos Falcó -las "500 viviendas". Por el contrario, encontramos porcentajes de abstención inferiores al $25 \%$ en los barrios Centro, Villacerrada y Parque Sur, todos ellos de elevados niveles socioeconómicos. La tesis de la distinción centroperiferia que apuntábamos como elemento de contraste en las páginas iniciales de este artículo parece revelarse como válida.

\section{Interpretación Estadística del Vínculo entre Variables Sociodemográficas y Datos Electorales}

En el diagrama de caja del Gráfico 1 podemos observar a los dos outliers que aparecen en la representación correspondiente a los porcentajes de abstención, y que corresponden a los dos barrios de menor nivel socioeconómico de la ciudad, La Estrella -que ofrece un 54'45\% de abstención- y La Milagrosa -con un 58'17\%. Los dos outliers señalados muestran valores extremos más allá del valor adyacente inferior (que, recordemos, recoge las puntuaciones que están a una distancia de 1'5 veces el rango intercuartílico del límite más cercano de la "caja", o cuartil más próximo). El mayor respaldo electoral hacia el Partido Popular, en unos comicios que -aun siendo de ámbito local- se encuentran muy profundamente afectados por el condicionante que representa un factor de ámbito internacional como la crisis económica, se plasma visualmente en un apoyo nítidamente superior a esta opción. La dispersión de sus niveles de apoyo electoral no difieren sustancialmente de los que encontramos en el PSOE, como muestran unas dimensiones de las correspondientes cajas, unas desviaciones típicas y unos rasgos intercuartílicos de magnitudes similares en todos los casos; a pesar de ello, sí que encontramos, en el caso del voto al Partido Popular una mayor distancia entre los valores extremos recogidos en los barrios de la ciudad, que oscilan -de una manera territorialmente muy significativa- entre el 65'79 conseguido en el barrio Centro y el muy escaso 25’33 del barrio de La Milagrosa. Las representaciones gráficas en forma de diagramas de caja facilitan la 
visualización de esta realidad. Las líneas que, partiendo verticalmente de la "caja", alcanzan a los valores adyacentes superior e inferior del voto PP tienen una mayor longitud que las correspondientes al voto PSOE. Las asimetrías que pueden observarse en el gráfico permiten inferir la inexistencia de una normalidad perfecta en las distribuciones aunque, como veremos, ello no invalida la utilización de los modelos estadísticos escogidos.

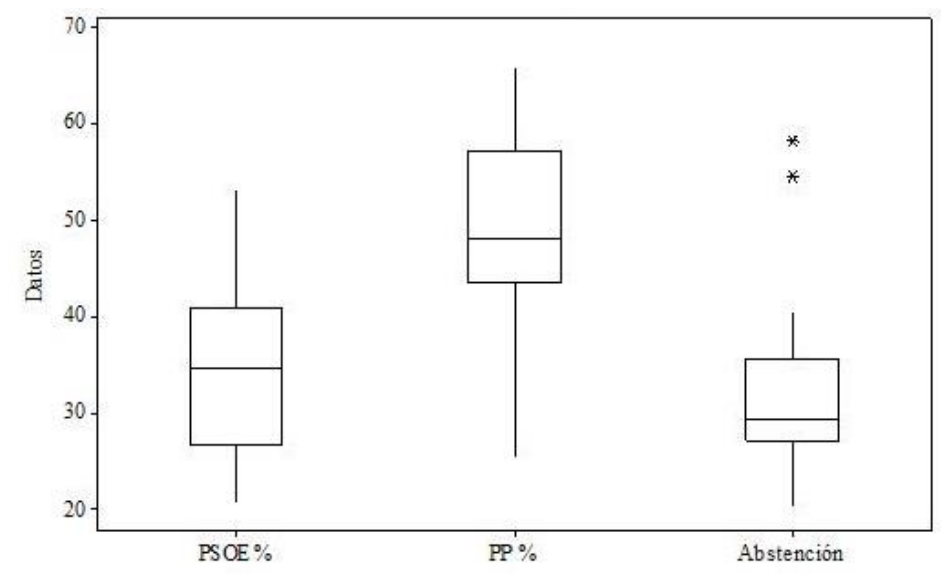

Fuente: elaboración propia a partir de datos del Ministerio del Interior, 2011.

Figura 1. Diagramas de caja de PSOE, PP y Abstención

De cara a verificar $-o$, en su caso, descartar- que las variables sociodemográficas mencionadas aportan una clave explicativa del comportamiento electoral de la población residente en los diversos entornos urbanos de nuestra ciudad, hemos optado por realizar unos análisis de regresión lineal. La opción de llevar a cabo unos análisis de correlación, con un índice de Pearson o similar, no parece en principio razonable, puesto que no se trata de que las variables sociodemográficas escogidas y el comportamiento electoral interaccionen entre sí -las variables, ciertamente, condicionan la actitud de los ciudadanos ante las urnas, pero no puede decirse, a la inversa, que los resultados electorales condicionen o estén a la 
base del nivel educativo, de edad o socioeconómico-. La idea es considerar que estos tres factores puedan ser utilizados como variables explicativas o independientes del comportamiento electoral, el cual debe entenderse, pues, como variable respuesta o dependiente. A su vez, dentro de la pluralidad de posibilidades analíticas que podemos encontrar en la "familia" de análisis de regresión, una opción razonable es utilizar un análisis de regresión múltiple, que nos indicara el peso que cada uno de nuestros factores sociodemográficos puede tener como condicionante del comportamiento electoral. Aunque entendemos que ésta es una decisión válida, existe una sospecha de multicolinealidad entre las variables independientes. Por ello, hemos incluido en el análisis los índices VIF (Variance Inflation Factor), que nos han permitido concluir la validez del modelo. Junto a ello, optamos por visualizar el vínculo entre las variables sociodemográficas utilizadas y voto a través de análisis de regresión lineal bivariante.

Para este tipo de modelo estadístico, es condición indispensable, de un lado, que los llamados "residuos" tengan una media de valor cero y una varianza constante, y, de otro, que los residuos estén normalmente distribuidos. El procedimiento habitual para corroborar estos hechos se lleva a cabo mediante el uso de gráficos de residuos (residual plots), en el caso de la primera condición mencionada, mientras que la segunda condición sería verificada a través de de representaciones gráficas de la probabilidad normal de los residuos de las puntuaciones. Probablemente convenga recordar, a estos efectos, que los modelos de regresión lineal incluyen una función que define la línea en torno a la cual los puntos de un gráfico de dispersión están distribuidos. Los llamados "residuos" son las diferencias entre cada uno de los valores y la citada línea o curva de ajuste. Los residual plots, para satisfacer la condición mencionada, no deben mostrar ninguna pauta homogénea de distribución de los puntos, que han de aparecer localizados de una manera aleatoria alrededor de la línea que representa el 0; en lo que se refiere a los gráficos de probabilidad normal de los residuos, los puntos han de aparecer ubicados en torno a la línea de ajuste. Hemos comprobado de esta manera la validez de nuestro modelo, aunque, en aras de no sobrecargar el artículo con información que pudiera ser considerada como superflua, no incluiremos las numerosas representaciones gráficas generadas. 


\section{Roblizo-Colmenero - La Configuración Espacial de la Ciudad}

Observaremos los resultados de nuestro análisis de regresión lineal bivariante mediante el uso de indicadores estadísticos; el primero de ellos será la $\mathrm{R}^{2}$, expresión matemática de la relación entre las variables explicativas indicadas y las variables dependientes, que en nuestro caso, recordemos, son los datos electorales expresados en puntuaciones $\mathrm{Z}$. El hecho de que estén expresados de esa manera ofrece, de cara al análisis de regresión lineal, resultados equiparables a los que arrojarían si estuviesen expresados porcentualmente -por la propia lógica matemática de estos indicadores-, pero el uso de los valores $\mathrm{Z}$ otorga una homogeneidad con las formas numéricas de las variables sociodemográficas utilizadas. Junto a ello, recurrimos a los valores p para tratar de, en su caso, corroborar las apreciaciones procedentes de los otros indicadores estadísticos evaluando la significatividad estadística de nuestras observaciones.

Los indicadores estadísticos $\mathrm{R}^{2}$ que vemos en la tabla 3 vienen a ser una plasmación en cifras de unos vínculos significativamente elevados, en el caso de los factores "educativo" y "socioeconómico", con los indicadores electorales, y con una relación real, aunque más débil, en el caso de "envejecimiento". La magnitud de las distintas cifras nos revela esta realidad. Por su parte, la interpretación estadística de los valores p se sustenta en el establecimiento de una hipótesis nula (H0), según la cual las distintas variables sociodemográficas seleccionadas no tienen ningún efecto sobre los índices de voto PSOE, PP y de abstención. La interpretación de este tipo de indicadores se lleva a cabo mediante las convenciones comúnmente utilizadas. En este caso, todos los valores p correspondientes a los factores educativo y socioeconómico son 0'000, lo que viene a indicar que hay una fuerte evidencia estadística en contra de la hipótesis de que las variables sociodemográficas seleccionadas no tienen ningún efecto sobre el voto PSOE y PP y sobre la abstención. En el caso de los valores p correspondientes a "envejecimiento", la convenciones interpretativas de la estadística indicarían una misma conclusión a la anteriormente mencionada, con fuerte evidencia en contra de la hipótesis nula, en lo referente a “abstención" y "voto PP”, mientras que en el caso del voto PSOE, el valor $\mathrm{p}=0$ '038 se encontraría entre 0'01 y 0'05, por lo que cabría interpretarlo en base a una moderada evidencia en contra de la hipótesis planteada. 
Aparece, por lo tanto, un cierto matiz estadístico que determina un cambio en la escala, aunque con una conclusión igualmente coincidente en el sentido de rechazar el que las variables indicadas no tengan ningún efecto sobre los datos electorales que hemos utilizado. De esta manera, tanto las puntuaciones $\mathrm{p}$ como los índices $\mathrm{R}^{2}$ aportan unas conclusiones que podemos considerar coincidentes en cuanto al vínculo entre los factores explicativos señalados y las principales opciones de voto.

Tabla 3.

Indicadores de regresión (puntuaciones $Z$ )

\begin{tabular}{|c|c|c|c|c|c|c|}
\hline & \multicolumn{2}{|c|}{ F. educativo } & \multicolumn{2}{|c|}{ F. socioeconómico } & \multicolumn{2}{|c|}{ Envejecimiento } \\
\hline & $\mathbf{R}^{2}$ & $\mathbf{P}$ & $\mathbf{R}^{2}$ & $\mathbf{P}$ & $\mathbf{R}^{2}$ & $\mathbf{P}$ \\
\hline PSOE & $73^{\prime} 4$ & 0’000 & $75 ’ 2$ & 0’000 & $20 ’ 8$ & 0’038 \\
\hline PP & 79’0 & 0’000 & 73 ' 8 & 0’000 & $32 ’ 3$ & 0’007 \\
\hline Abstención & $89 ’ 0$ & 0’000 & $84^{\prime} 6$ & 0’000 & $37^{\prime} 8$ & 0’003 \\
\hline
\end{tabular}

Fuente: elaboración propia a partir de datos del Observatorio Municipal de Igualdad de Oportunidades de Albacete y del Ministerio del Interior, 2011

Desde otra perspectiva, si llevamos a cabo un análisis de regresión múltiple que nos indique el peso de cada una de las variables sociodemográficas consideradas en las distintas opciones de voto utilizadas, encontramos los resultados que recogemos en las tablas 4, 5 y 6. La lógica subyacente cuando usamos esta metodología radica en que aportamos una estimación del cambio en cada una de las variables dependientes utilizadas (voto PSOE, voto PP y abstención) cuando se produce un cambio en una variable independiente (factor educativo, factor socioeconómico o envejecimiento) y el resto de ellas se mantiene constante (Berry y Sanders, 2000). En las tablas 4, 5 y 6 podemos ver como las puntuaciones VIF (Variance Inflation Factor) son inferiores a 10, por lo que puede concluirse que no hay razón para estimar que existe colinealidad (Bowerman y O’Connell, 1990).

Las puntuaciones $\mathrm{p}$ se sitúan, en lo que se refiere a los factores educativos y socioeconómico, por encima de 0'05, lo que hace que haya que adoptar cierta precaución a la hora de concluir que tales factores puedan tener una incidencia determinante; sin embargo -a excepción de la 
puntuación correspondiente al factor socioeconómico en el voto PP- no exceden el valor 0'1, por lo que podríamos asumir, con la precaución reseñada, su valor analítico. En el caso de "envejecimiento", las puntuaciones $\mathrm{p}$ exceden ampliamente el 0'05. Una lógica similar se observa en las ecuaciones de regresión, en las que los factores educativos y socioeconómicos, en cada una de las opciones de voto consideradas, muestran una incidencia apreciable, mientras que la variable envejecimiento registra una capacidad predictiva ciertamente mínima. Realmente, esta variable debería ser eliminada del modelo, pero hemos querido mantener los datos referentes a ella para visualizar el carácter marginal que presenta como factor de influencia en el comportamiento electoral.

En todo caso, los valores de $\mathrm{R}^{2}$, que indican el porcentaje de variación que explican las variables independientes utilizadas, son elevados, lo que permite inferir la validez de los modelos explicativos. Las puntuaciones $\mathrm{T}$, como cabría esperar, ofrecen valores mayores a 2 o menores a -2 cuando encontramos una significatividad estadística al nivel 0’05.

Tabla 4.

Análisis de regresión: Z PSOE versus Envejecimiento, Factor educativo y Factor socioeconómico

\begin{tabular}{lccccc}
\hline Predictor & Coef & SE Coef & T & P & VIF \\
\hline Constante & $-0,0697$ & 0,1006 & $-0,69$ & 0,498 & \\
Envejecimiento & 0,1447 & 0,1344 & 1,08 & 0,297 & 1,8 \\
Factor educativo & 0,4734 & 0,2704 & 1,75 & 0,098 & 7,1 \\
F. socioeconómico & 0,6760 & 0,3802 & 1,78 & 0,093 & 6,1 \\
\hline
\end{tabular}

La ecuación de regresión es Z PSOE $=-0,070+0,145$ Envejecimiento + 0,473 Factor educativo + 0,676 F. socioeconómico

$\mathrm{S}=0,457020 \quad \mathrm{R}-\mathrm{Sq}=79,1 \% \quad \mathrm{R}-\mathrm{Sq}(\operatorname{adj})=75,5 \%$

Fuente: elaboración propia a partir de datos del Observatorio Municipal de Igualdad de Oportunidades de Albacete y del Ministerio del Interior, 2011. 
Tabla 5 .

Análisis de regresión: $Z$ PP versus Envejecimiento, Factor educativo y Factor socioeconómico

\begin{tabular}{lccccc}
\hline Predictor & Coef & SE Coef & T & P & VIF \\
\hline Constante & 0,0332 & 0,1047 & 0,32 & 0,755 & \\
Envejecimiento & $-0,0083$ & 0,1398 & $-0,06$ & 0,954 & 1,8 \\
Factor educativo & $-0,6207$ & 0,2813 & $-2,21$ & 0,041 & 7,1 \\
F. socioeconómico & $-0,4264$ & 0,3955 & $-1,08$ & 0,296 & 6,1 \\
\hline
\end{tabular}

La ecuación de regresión es Z PP = 0,033 - 0,008 Envejecimiento - 0,621 Factor educativo $0,426 \mathrm{~F}$. socioeconómico

$\mathrm{S}=0,475449 \quad \mathrm{R}-\mathrm{Sq}=80,3 \% \quad \mathrm{R}-\mathrm{Sq}(\operatorname{adj})=76,9 \%$

Fuente: elaboración propia a partir de datos del Observatorio Municipal de Igualdad de Oportunidades de Albacete y del Ministerio del Interior, 2011.

Tabla 6.

Análisis de regresión: Z Abs. versus Envejecimiento, Factor educativo y Factor socioeconómico

\begin{tabular}{lccccc}
\hline Predictor & Coef & SE Coef & T & P & VIF \\
\hline Constante & 0,06286 & 0,07165 & 0,88 & 0,393 & \\
Envejecimiento & $-0,01314$ & 0,09571 & $-0,14$ & 0,892 & 1,8 \\
Factor educativo & 0,6118 & 0,1926 & 3,18 & 0,006 & 7,1 \\
F. socioeconómico & 0,5353 & 0,2707 & 1,98 & 0,064 & 6,1 \\
\hline
\end{tabular}

La ecuación de regresión es Z Abs. = 0,0629 - 0,0131 Envejecimiento + 0,612 Factor educativo + 0,535 F. socioeconómico $\mathrm{S}=0,325473 \quad \mathrm{R}-\mathrm{Sq}=91,1 \% \quad \mathrm{R}-\mathrm{Sq}(\operatorname{adj})=89,5 \%$

Fuente: elaboración propia a partir de datos del Observatorio Municipal de Igualdad de Oportunidades de Albacete y del Ministerio del Interior, 2011.

\section{Conclusiones}

Ciertamente, el entorno urbano se sigue mostrando, como ya indicaran algunos clásicos, como un contexto en el que es muy posible hallar regularidades interpretativas y homogeneidades significativas de cara al análisis de diversas formas de comportamiento humano -entre ellas, como hemos tratado de observar, el político-. Las configuraciones espaciales siguen mostrando continuidades fruto de la ubicación espacial en base a los 


\section{Roblizo-Colmenero - La Configuración Espacial de la Ciudad}

distintos niveles de poder adquisitivo, con las connotaciones sociodemográficas que ello conlleva.

De esta manera, hemos observado - a través del espacio urbano- el sólido vínculo existente entre condición socioeconómica y comportamiento electoral, tanto en lo referente a las opciones políticas más principales y representativas de los dos polos del espacio político español como en lo relativo a la participación electoral. Todavía la clásica distinción centroperiferia muestra su utilidad -en su doble vertiente social, según acuñara Milbrath, y, con ciertos matices, urbana- como variable de incidencia en la afluencia del electorado a las urnas. Específicamente, el factor educativo se ha considerado -y, ciertamente, demuestra serlo- especialmente significativo en este sentido, puesto que los mayores niveles suelen implicar una mayor cercanía a los temas públicos y, en consecuencia, una mayor proximidad también a los asuntos políticos. Pero, además, hemos apreciado también la vinculación de este factor con aspectos que no se limitan a la participación y que remiten también a la orientación hacia las dos opciones más representativas. En todo caso, tanto los indicadores estadísticos referentes al factor educativo como al socioeconómico sugieren que esa vinculación no debe asumirse de una manera que pudiéramos considerar determinista.

El grado de envejecimiento de la población, por su parte, podría considerarse ligado al nivel cultural, en tanto las generaciones mayores tuvieron más dificultades en el acceso a la educación que las más jóvenes, y viceversa. Sin embargo, la ubicación en el espacio urbano hace que esa interrelación aparezca difuminada, por la incidencia del acceso a la vivienda en los distintos grupos sociales, que puede haber determinado que grupos de avanzada edad se encuentren ubicados en áreas muy favorables, con independencia de sus niveles culturales o económicos actuales. Los avatares que, a lo largo de las últimas décadas, han experimentado los precios de la vivienda contribuyen, sin duda, a dar al envejecimiento un carácter más intangible como factor explicativo.

Los factores educativo y socioeconómico seleccionados manifiestan su relevancia como factores explicativos incluso en un contexto electoral muy convulso $-\mathrm{y}$, por lo tanto, cambiante-. Se da así cabida a entender que el llamado modelo de identificación de partido muestra su vigencia en este entorno urbano. A pesar de haber usado variables de clara carga estructural, 
es posible reclamar también, con toda nitidez, la vigencia de planteamientos basados en lógica de la acción colectiva o de la elección racional. Y ello es así porque parece que no es difícil encontrar vínculos basados en el cálculo de intereses en las elecciones que mayoritariamente hacen cada uno de los sectores sociales analizados, tanto en lo referente a opciones entre distintas candidaturas como a participación en el proceso electoral.

De esta forma, la pervivencia del territorio urbano como factor definitorio de la diferenciación social, a la manera de lo que fuera formulado por la Escuela de Chicago, encuentra su concreción en el comportamiento electoral, si tomamos los indicadores básicos de mayor capacidad definitoria. En definitiva, la ciudad se nos ha revelado, una vez más, como un elemento de notable utilidad práctica como laboratorio en el que poder hacer un trabajo de observación de la realidad electoral, con la diversidad de implicaciones sociológicas que ello conlleva. Y ello acontece, nos atreveríamos a decir, con unas conclusiones con potencialidades capaces de transcender lo observado. Nuestro pequeño experimento social -por parafrasear a la Escuela de Chicago- de laboratorio, como tal, siempre será susceptible de ir más allá de sí mismo.

\section{Notas}

${ }^{1}$ based on the idea that 'birds of a feather flock together', it gives recognition to the fact that people with broadly similar economic, social and lifestyle characteristics tend to congregate in particular neighbourhoods and exhibit similar patterns of purchasing behaviour and outlook (Traducción propia del autor).

${ }^{2}$ The city, in short, shows the good and evil in human nature in excess. It is this fact, more than any other, which justifies the view that would make of the city a laboratory or clinic in which human nature and social processes may be most conveniently and profitably studied (Traducción propia del autor).

\section{Referencias}

Berry, W., y Sanders, M. (2000). Understanding multivariate research. Boulder: Westview Press.

Busteed, M. (1975). Geography and voting behavior. Oxford: Oxford University Press. 
Bowerman, B., y O'Connell, R. (1990). Linear statistical models: and applied approach. Belmont: Duxbury.

Cox, K. (1969). The voting decision in spatial context. En C. Board, R. Chorley, P. Hagget y D. Stoddart (Eds.), Progress in Human Geography, vol. 1. London: Edward Arnold.

Downs, A. (1973). Teoría económica de la democracia. Madrid: Aguilar. Green, D., Palmquist, B., y Schickler, E. (2004). Partisan hearts and minds. New Haven: Yale University Press.

Harrop, M., y Miller, W. (1987). Elections and voters. A comparative introduction. New York: New Amsterdam Books.

Izquierdo, J. (1984). Las elecciones de la transición en Castilla-La Mancha: Albacete. Albacete: Instituto de Estudios Albacetenses.

Lewis-Beck, M., Norpoth, H., Jacoby, W., y Weisberg, H. (2008). The American voter revisited. Michigan: University of Michigan Press.

Lipset, S. (1987). El hombre político. Las bases sociales de la política. Madrid: Tecnos.

Milbrath, L. (1965). Political participation. Chicago: Rand McNally. Mitchel, R., Dorling, D., y Shaw, M. (2000). Inequalities in life and death. Bristol: Policy Press/Joseph Rowntree Foundation.

Observatorio Municipal de Igualdad de Oportunidades de Albacete (2011).

Barrios de Albacete: igualdad, situación social y recursos.

Albacete: Ayuntamiento de Albacete.

Park, R. (1952). The city: suggestions for the investigation of human behaviour in the urban environment. American Journal of Sociology, 20, 577-612.

Taylor, P., y Johnson, R. (1979). Geography of elections. Londres: Penguin. Wilson, R., Gilligan, C., y Pearson, D. (1992). Strategic Marketing Management. Londres: Heinemann-Butterworth.

Manuel Jacinto Roblizo-Colmenero es profesor en el Departamento de Filosofía de la Universidad de Castilla-La Mancha

Dirección de contacto: Departamento de Filosofía, Antropología, Sociología y Estética. Facultad de Educación. Campus de Albacete. Pza de la Universidad, 3 -02071- Albacete, España. Email: Manuel.Roblizo@uclm.es 